\title{
Camera settings and habitat type influence the accuracy of citizen science approaches to camera trap image classification.
}

Nicole Egna ${ }^{1}$, DAVID O'CONNOR ${ }^{2}$, Jenna Stacy-Dawes ${ }^{2}$, Mathias Tobler ${ }^{2}$, Nicholas Pilfold $^{2}$, Kristin Neilsen ${ }^{2}$, Brooke Simmons ${ }^{3}$, Elizabeth Davis ${ }^{2}$, Mark Bowler ${ }^{4}$, Symon Masiaine $^{2}$, Daniel Lenaipa ${ }^{5}$, Jonathan Lenyakopiro ${ }^{5}$, Lexson Larpei ${ }^{6}$, Johnson Lekushan ${ }^{5}$, Ruth Lekupanai $^{7}$, Jesus Lekalgitele ${ }^{5}$, Joseph Lemirgishan ${ }^{5}$, Lekuran Lemingani ${ }^{5}$, Ranis Lesipiti $^{5}$, Masenge Lororua ${ }^{5}$, Sebastian Rabhayo ${ }^{5}$, Kirstie Ruppert ${ }^{2}$, Jenny Glikman ${ }^{2}$, Julian Fennessy $^{8}$, Arthur Muneza ${ }^{8}$, and Megan Owen ${ }^{2}$

${ }^{1}$ Duke University Nicholas School of the Environment

${ }^{2}$ San Diego Zoo Institute for Conservation Research

${ }^{3}$ Lancaster University Department of Physics

${ }^{4}$ University of Suffolk

${ }^{5}$ Namunyak Wildlife Conservation Trust

${ }^{6}$ Loisaba Conservancy

${ }^{7}$ Namunyak Conservation Wildlife Trust

${ }^{8}$ Giraffe Conservation Foundation

May 6, 2020

\begin{abstract}
Scientists are increasingly using volunteer efforts of citizen scientists to classify images captured by motion-activated trailcameras. The rising popularity of citizen science reflects its potential to engage the public in conservation science and accelerate processing of the large volume of images generated by trail-cameras. While image classification accuracy by citizen scientists can vary across species, the influence of other factors on accuracy are poorly understood. Inaccuracy diminishes the value of citizen science derived data and prompts the need for specific best practice protocols to decrease error. We compare the accuracy between three programs that use crowdsourced citizen scientists to process images online: Snapshot Serengeti, Wildwatch Kenya, and AmazonCam Tambopata. We hypothesized that habitat type and camera settings would influence accuracy. To evaluate these factors, each photo was circulated to multiple volunteers. All volunteer classifications were aggregated to a single best answer for each photo using a plurality algorithm. Subsequently, a subset of these images underwent expert review and were compared to the citizen scientist results. Classification errors were categorized by the nature of the error (e.g. false species or false empty), and reason for the false classification (e.g. misidentification). Our results show that Snapshot Serengeti had the highest accuracy (97.9\%), followed by AmazonCam Tambopata (93.5\%), then Wildwatch Kenya (83.4\%). Error type was influenced by habitat, with false empty images more prevalent in open-grassy habitat (27\%) compared to woodlands (10\%). For medium to large animal surveys across all habitat types, our results suggest that to significantly improve accuracy in crowdsourced projects, researchers should use a trail-camera set up protocol with a burst of three consecutive photos, a short field of view, and consider appropriate camera sensitivity. Accuracy level comparisons such as this study can improve reliability of future citizen science projects, and subsequently encourage the increased use of such data.
\end{abstract}

\section{Hosted file}

Main Document.pdf available at https://authorea.com/users/318534/articles/448416-camera- 
settings-and-habitat-type-influence-the-accuracy-of-citizen-science-approaches-tocamera-trap-image-classification 